\title{
Enhanced Subspaced Approach to Interference Mitigation
}

\author{
Pei Xiao $^{\dagger}$, Wu Yin ${ }^{\dagger \dagger}$, Rahim Tafazolli ${ }^{\dagger}$, Sherif Welsen Shaker ${ }^{\dagger \dagger}$ \\ ${ }^{\dagger}$ Centre for Communication Systems Research (CCSR) \\ University of Surrey, Guildford, Surrey, GU2 7XH, United Kingdom \\ E-mail: $\{$ P.Xiao,R.Tafazollia\}@surrey.ac.uk \\ ${ }^{\dagger \dagger}$ Research and Development, Kuang-Chi Institute of Advanced Technology \\ High-Tech Industrial Estate, Nanshan District, Shenzhen 518000, China \\ E-mail: \{wuyin, welsen\}@kuang-chi .org
}

\begin{abstract}
In this paper, we study an enhanced subspace based approach for the mitigation of multiple access interference (MAI) in direct-sequence code-division multiple-access (DS-CDMA) systems over frequency-selective channels. Blind multiuser detection based on signal subspace estimation is of special interest in mitigating MAI in CDMA systems since it is impractical to assume perfect knowledge of parameters such as spreading codes, time delays and amplitudes of all the users in a rapidly changing mobile environment. We develop a new blind multiuser detection scheme which only needs the priori knowledge of the signature waveform and timing of the user of interest. By exploiting the improper nature of multiple access interference (MAI) and intersymbol interference (ISI), the enhanced detector shows clear superiority to the conventional subspace-based blind multiuser detector. The performance advantages are shown to be more obvious in heavily loaded systems when the number of active users is large.
\end{abstract}

\section{INTRODUCTION}

It is well known that CDMA communication systems are interference limited, that is, the system capacity is limited by the multiple access interference (MAI) [1]. In the recent decade, there have been significant efforts in developing multiuser detection technologies to overcome the MAI problem. The maximum likelihood sequence estimator is the optimum detector in terms of bit error rate (BER) performance [1], but its complexity grows exponentially with the number of users, which is prohibitive for practical implementation. Linear multiuser detectors, such as the decorrelating detector [2,3] and the minimum mean square error (MMSE) detector [4], are suboptimum solutions, but they have low complexity, which only grows linearly with the number of users. An adaptive MMSE detector was introduced in [5] based on training sequence and the stochastic gradient descent training algorithm. The need for training sequence is further avoided in the blind multiuser detection scheme proposed in [6], where a blind MMSE detector is developed by minimizing the mean output energy (MOE) subject to an orthogonality constraint. Another approach to blind detection is based on subspace estimation. In [7,8], the decorrelating detector and the MMSE detector are expressed in closed forms of the signal subspace components, and it was shown that compared with the previous MOE blind multiuser detector, the proposed subspace-based blind multiuser detector offers lower computational complexity, better performance and robustness against signature waveform mismatch.
In practical high-speed wireless communications, the intersymbol interference (ISI) caused by multipath propagation imposes significant concern. One way to overcome the effect of ISI is to employ multicarrier technique to transform a frequency selective fading channel to flat fading channels [9]. However, since the multipath signals become non-resolvable, the multicarrier approaches forgo the opportunity to make use of the effective multipath diversity gain. RAKE receiver is commonly used to solve the multipath problem [10], but it is optimum only in single-user systems. For multiuser systems, the RAKE structure is still applicable but the matched filters in the front end are replaced by linear multiuser detectors [11]. In [12], the ISI is modelled as virtual MAI and detectors in expanded subspace are derived to simultaneously suppress MAI and ISI. The expanded detectors are not limited by the channel length, meanwhile, they maintain the desirable properties of distributed implementation without detection delays.

It has been shown that under certain conditions, the performance of linear multiuser receivers can be significantly improved if not only the received signal, but also its complex conjugate is processed. The resulting schemes are commonly referred to as widely linear (WL) receivers. The application of widely linear processing (WLP) was first developed for improving performance of DS-CDMA systems with improper data modulation, such as pulse-amplitude modulation (PAM), offset quadrature phase shift keying (OQPSK), offset quadrature amplitude modulation (OQAM), and binary phase-shift-keying (BPSK), etc.. For example, it was shown in [13] that for systems employing BPSK modulation, full exploitation of the available information on the second-order statistics of the observations entails the use of WLP, and the resulting scheme outperforms the other schemes currently known in the literature. It was shown in [14] that OQPSK modulation generates improper complex MAI and the application of WLP to the OQPSK modulated CDMA systems yields remarkable output signal-to-noise ratio (SNR) gains over linear receivers. Similar conclusions were made for a WL MOE receiver derived in [15]. The application of WLP to the complex modulation schemes was addressed in several papers, e.g., in $[16,17]$ where WLP was applied to complex signals which become improper by space-time block coding or widely linear space-time mapping. A novel iterative multiuser detector for DS-CDMA systems with complex modulation schemes was proposed in [18]. 
Due to the fact that the residual MAI becomes improper when soft decision feedback is used to cancel the MAI, the employment of WLP achieves significant gains in power efficiency and improves convergence speed.

In this paper, we further develop the algorithm presented in $[7,8]$ and derive a blind multiuser detector using the subspace components derived from both the autocorrelation and pseudo-autocorrelation matrices of observations. Results show that by exploiting the additional information contained in the pseudo-autocorrelation matrix and by utilizing the improperness of the MAI and ISI, a noticeable performance gain can be attained compared to the conventional blind detector previously proposed in the literature. The rest of the paper is organized as follows. The system model is presented in Section II. The conventional blind detection scheme based on subspace approach is briefly reviewed, and an improved blind detector is developed in Section III. Section IV presents the simulation results, and finally, the conclusions are drawn in Section V.

Throughout this paper, we adopt the following notations: $(\cdot)^{\mathcal{H}}$ matrix conjugate transpose, $(\cdot)^{\mathcal{T}}$ matrix transpose, $(\cdot)^{*}$ matrix conjugate, $\mathrm{E}[\cdot]$ expectation operation, $\mathbb{C}$ complex filed.

\section{SySTEM MODEL}

Consider a DS-CDMA system employing BPSK modulation to transmit signals through multipath channels. The symbol interval and chip interval are denoted by $T$ and $T_{c}$, respectively. The $k$ th user's spreading waveform is

$$
c_{k}(t)=\sum_{n=0}^{N-1} c_{k}(n) \psi\left(t-n T_{c}\right), t \in[0, T], k=1, \ldots, K
$$

where $K$ denotes the number of users; $N=T / T_{c}$ is the spreading factor; $\left\{c_{k}(n)\right\}_{n=0}^{N-1}$ is the normalized signature code assigned to the $k$ th user; $\psi(t)$ is a normalized chip waveform defined in $\left[\begin{array}{ll}0 & T_{c}\end{array}\right]$. The multipath channel is modeled by a tapped delay line with tap spacing $1 / W[11]$ ( $W$ is the bandwidth of the spread-spectrum signals) and tap coefficients $\left\{h_{k}(l)\right\}_{l=0}^{L-1}$, where $L$ is the number of resolvable paths for each user. We consider the case when $W=1 / T_{c}, L=\left\lceil T_{m} / T_{c}\right\rceil$, where $T_{m}$ is the multipath delay spread, and $\lceil x\rceil$ denotes the value obtained by rounding $x$ to the nearest integer which is greater or equal to $x$. Transmitting the signals through the multipath channel, the received signal due to the $k$ th user is given by

$$
y_{k}(t)=\sum_{i=-\infty}^{\infty} A_{k} b_{k}(i) \sum_{l=0}^{L-1} h_{k}(l) c_{k}\left(t-i T-l / W-\tau_{k}\right)
$$

where $A_{k}, b_{k}(i)$ and $\tau_{k}$ are the amplitude, the $i$ th transmitted information bit, and the transmission delay of the $k$ th user, respectively; $b_{k}(i) \in\{+1,-1\}$ follows identical independent distribution (i.i.d.). For simplicity, the detectors in this paper are derived for the case when $\tau_{k}=0$. The total received signal is the superposition of the information-bearing data signals of $K$ users plus the additive white Gaussian noise (AWGN), i.e., $r(t)=\sum_{k=1}^{K} y_{k}(t)+v(t)$, where $v(t)$ is the zero-mean white Gaussian noise with variance $\sigma^{2}$. After chip-matched filtering followed by chip-rate sampling, the discrete-time format of the received signal is given by

$$
r(j)=\sum_{k=1}^{K} y_{k}(j)+v(j)
$$

where

$$
\begin{aligned}
y_{k}(j) & =\sum_{i=-\infty}^{\infty} A_{k} b_{k}(i) s_{k}(j-i N) \\
s_{k}(n) & =\sum_{l=0}^{L-1} h_{k}(l) c_{k}(n-l) ; \\
v(j) & =\int_{j T_{c}}^{(j+1) T_{c}} v(t) \psi\left(t-j T_{c}\right) d t .
\end{aligned}
$$

On the transmitter side, the energy of each symbol is limited in duration $N T_{c}$. However, on the receiver side, the energy is spread over an extended interval $(N+L-1) T_{c}$ due to the channel convolution effect, which can be observed from (2). We aim to design a multiuser detector that detects the signals in a symbol-by-symbol fashion and employ a processing window of length $N+L-1$ to model the received signal in a vector form. Falling within the window are not only the information bits for the current time instant, but also those before and after the current time instant. The received signal vector thus contains ISI from the past as well as the future symbols. In order to describe it quantitatively, we define $P=\lceil(L-1) / N\rceil$. Then, $2 P+1$ symbols from the desired user are included in the processing window of length $N+L-1$, including the current symbol, $P$ symbols before and $P$ symbols after the current symbol. Taking into account of all the $\mathrm{K}$ users, there are $(2 P+1) K$ symbols involved in detecting one symbol of the desired user. The $(N+L-1)$-element received signal vector can be expressed as

$$
\mathbf{r}(i)=\sum_{k=1}^{K} \mathbf{y}_{k}(i)+\mathbf{v}(i)
$$

where

$$
\begin{aligned}
& \mathbf{y}_{k}(i)=A_{k} b_{k}(i) \mathbf{s}_{k}+\sum_{j=1}^{P} A_{k} b_{k}(i-j) \underline{\mathbf{s}_{k, j}}+\sum_{j=1}^{P} A_{k} b_{k}\left(i+j \underline{\underline{\mathbf{s}_{k, j}}}\right. \\
& \mathbf{s}_{k}=\left[\begin{array}{lll}
s_{k}(0) & \ldots & s_{k}(N+L-2)
\end{array}\right]^{\mathcal{T}} ; \\
& \underline{\mathbf{s}_{k, j}}=\left[\begin{array}{lllllll}
s_{k}(j N) & s_{k}(j N+1) & \ldots & s_{k}(N+L-2) & 0 & \ldots & 0
\end{array}\right]^{\mathcal{T}} \\
& \underline{\underline{\mathbf{s}_{k, j}}}=\left[\begin{array}{lllllll}
0 & \ldots & 0 & s_{k}(0) & s_{k}(1) & \ldots & s_{k}(N+L-2-j N)
\end{array}\right]^{\mathcal{T}} \text {; } \\
& \mathbf{v}(i)=\left[\begin{array}{lll}
v(i N) & \ldots & v(i N+N+L-2)
\end{array}\right]^{\mathcal{T}} \text {. }
\end{aligned}
$$

Define

$$
\begin{aligned}
& \underline{\mathbf{s}_{k}}=\left[\begin{array}{lll}
\underline{\mathbf{s}_{k, 1}} & \cdots & \underline{\mathbf{s}_{k, P}}
\end{array}\right] ; \quad \underline{\underline{\mathbf{s}_{k}}}=\left[\begin{array}{lll}
\underline{\underline{\mathbf{s}_{k, 1}}} & \cdots & \underline{\underline{\mathbf{s}_{k, P}}}
\end{array}\right] ; \\
& \mathbf{S}=\left[\begin{array}{llllllllll}
\mathbf{s}_{1} & \underline{\mathbf{s}_{1}} & \underline{\underline{\mathbf{s}_{1}}} & \mathbf{s}_{2} & \underline{\mathbf{s}_{2}} & \underline{\underline{\mathbf{s}_{2}}} & \ldots & \mathbf{s}_{K} & \underline{\mathbf{s}_{K}} & \underline{\underline{\mathbf{s}_{K}}}
\end{array}\right]^{\mathcal{T}} ; \\
& \mathbf{A}=\operatorname{diag}\{\underbrace{A_{1} \ldots A_{1}}_{2 P+1} \underbrace{A_{2} \ldots A_{2}}_{2 P+1} \cdots \underbrace{A_{k} \ldots A_{k}}_{2 P+1}\} ;
\end{aligned}
$$

$$
\begin{aligned}
& \mathbf{b}_{k}(i)=\left[\begin{array}{lllllll}
b_{k}(i) & b_{k}(i-1) & \ldots & b_{k}(i-P) & b_{k}(i+1) & \ldots & b_{k}(i+P)
\end{array}\right] \\
& \mathbf{b}(i)=\left[\begin{array}{lll}
\mathbf{b}_{1}(i) & \ldots & \mathbf{b}_{K}(i)
\end{array}\right]^{\mathcal{T}},
\end{aligned}
$$

then (4) can be written in a compact form as

$$
\mathbf{r}(i)=\mathbf{S A} \mathbf{b}(i)+\mathbf{v}(i) .
$$

In (5), $b_{k}(i), b_{k}(i-j), b_{k}(i+j)$ are all from the $k$ th user. However, they can be regarded as statistically independent signals from different users which include the $k$ th user with modified signature codes $\mathbf{s}_{k}$, and $2 P$ virtual users with signature 
codes $\underline{\mathbf{s}}_{k, j}$ and $\mathbf{s}_{k, j}$. For the signal model expressed by (5), a

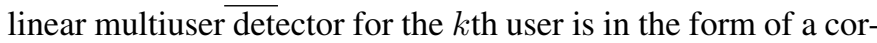
relator followed by a hard limiter $\hat{b}_{k}(i)=\operatorname{sgn}\left(\mathbf{m}_{k}^{\mathcal{H}} \mathbf{r}(i)\right)$, where $\mathbf{m}_{k} \in \mathbb{C}^{N+L-1}$.

\section{BLIND MULTIUSER DETECTORS}

We focus on blind detection schemes in this work, i.e., the detector $\mathbf{m}_{k}$ is derived only based on the parameters, e.g., the signature waveform of the desired user. Blind decorrelating detectors and blind MMSE detectors based on subspace estimation were introduced in [7,8]. It is well-known fact that decorrelating detectors have the noise enhancement problem and inferior performance to their MMSE counterparts, especially at how SNRs. For the purpose of this study, we will only consider blind MMSE detection schemes.

\section{A. Conventional blind MMSE detector}

Assume that user 1 is the user of interest. The linear MMSE multiuser detector $\mathbf{m}_{1}$ of user 1 minimizes the MSE

$$
\epsilon=\mathrm{E}\left[\left|A_{1} b_{1}-\mathbf{m}_{1}^{\mathcal{H}} \mathbf{r}\right|^{2}\right]
$$

subject to $\mathbf{m}_{1}^{\mathcal{H}} \mathbf{s}_{1}=1$. In order to derive the solution to (6), we perform eigen-decomposition of the autocorrelation matrix

$$
\begin{aligned}
\mathbf{C}_{\mathbf{r r}} & =\mathrm{E}\left[\mathbf{r r}^{\mathcal{H}}\right]=\left[\begin{array}{ll}
\mathbf{U}_{s} & \mathbf{U}_{n}
\end{array}\right]\left[\begin{array}{cc}
\Lambda_{s} & \mathbf{0} \\
\mathbf{0} & \Lambda_{n}
\end{array}\right]\left[\begin{array}{l}
\mathbf{U}_{s}^{\mathcal{H}} \\
\mathbf{U}_{n}^{\mathcal{H}}
\end{array}\right] \\
& =\mathbf{U}_{s} \Lambda_{s} \mathbf{U}_{s}^{\mathcal{H}}+\mathbf{U}_{n} \Lambda_{n} \mathbf{U}_{n}^{\mathcal{H}},
\end{aligned}
$$

where $\Lambda_{s}$ is a $(2 P+1) K \times(2 P+1) K$ diagonal matrix with positive diagonal elements, it contains the largest eigenvalues of $\mathbf{C}_{\mathbf{r r}}$ that are greater than $\sigma^{2}$, and $\mathbf{U}_{s}$ is $(N+L-1) \times(2 P+1) K$ matrix containing the corresponding orthonormal eigenvectors; $\Lambda_{n}=\sigma^{2} \mathbf{I}$ (where $\mathbf{I}$ is an identity matrix) contains eigenvalues that are equal to $\sigma^{2}$, and $\mathbf{U}_{n}$ contains the corresponding orthonormal eigenvectors. The columns of $\mathbf{U}_{s}$ span the signal subspace and the columns of $\mathbf{U}_{n}$ span the noise subspace. It can be shown that $\mathbf{S}$ and $\mathbf{U}_{s}$ have the same rank and span the same subspace. If $\mathbf{s}_{k}, \underline{\mathbf{s}_{k, j}}$ and $\mathbf{s}_{k, j}, k=1, \ldots, K$ are linearly independent, the signal subspace is $\mathbb{C}^{(N+L-1) \times(2 P+1) K}$, it is an expanded signal subspace compared to the signal subspace $\mathbb{C}^{N \times K}$ for AWGN channel. The solution to (6) is given by $[7,8]$

$$
\mathbf{m}_{1}=A_{1}^{2} \mathbf{U}_{s} \Lambda_{s}^{-1} \mathbf{U}_{s}^{\mathcal{H}} \mathbf{s}_{1}
$$

As shown by (7), the conventional blind MMSE detector can be expressed in closed form in terms of signal subspace components $\Lambda_{s}$ and $\mathbf{U}_{s}$, as well as the first user's effective signature waveform $\mathbf{s}_{1}$.

\section{B. Improved blind MMSE detector}

For a complex random vector $\mathbf{r}$, its second-order statistics are completely characterized by its autocorrelation matrix $\mathbf{C}_{\mathbf{r r}}=\mathrm{E}\left[\mathbf{r r}^{\mathcal{H}}\right]$ as well as the pseudo-autocorrelation matrix $\tilde{\mathbf{C}}_{\mathbf{r r}}=\mathrm{E}\left[\mathbf{r r}^{\mathcal{T}}\right]$ [19]. Most existing studies on receiver algorithms (including the one presented previously in Section III-A) only exploit the information contained in the autocorrelation function of the observed signal. The pseudo-autocorrelation matrix $\tilde{\mathbf{C}}_{\mathbf{r r}}$ is usually not considered and is implicitly assumed to be zero. While this is the optimum strategy when dealing with proper complex random processes (i.e., when pseudoautocorrelation $\tilde{\mathbf{C}}_{\mathbf{r r}}$ is vanishing) [20], it turns out to be suboptimum in situations where the transmitted signals and/or interference are improper complex random processes (i.e., $\tilde{\mathbf{C}}_{\mathbf{r r}}$ is non-vanishing), for which the performance of a linear receiver can generally be improved by the use of WLP [21]. Next, we show how the performance of a blind MMSE detector can be improved by applying WLP and by incorporating the pseudoautocorrelation function to the detection algorithm. For the improved blind detector, we re-define the MMSE criterion as follows

$$
\epsilon^{\prime}=\mathrm{E}\left[\left|\operatorname{Re}\left\{\mathbf{m}_{1}^{\mathcal{H}} \mathbf{r}\right\}-A_{1} b_{1}\right|^{2}\right]
$$

subject to $\operatorname{Re}\left\{\mathbf{m}^{\mathcal{H}} \mathbf{s}_{1}\right\}=1$. The reasoning for this modification is that a conventional MMSE filter yields a complex valued filter output. However, only the real part of this output is relevant for the decision in a system with real valued constellation. Minimization of (8) will result in a better estimator than the one designed under the criterion expressed by (6) since $\epsilon^{\prime}<\epsilon$ [22]. Note that

$$
\begin{aligned}
\operatorname{Re}\left\{\mathbf{m}^{\mathcal{H}} \mathbf{r}\right\} & =\frac{1}{2}\left[\mathbf{m}^{\mathcal{H}} \mathbf{r}+\left(\mathbf{m}^{\mathcal{H}} \mathbf{r}\right)^{*}\right]=\frac{1}{2}\left[\mathbf{m}^{\mathcal{H}} \mathbf{r}+\mathbf{m}^{\mathcal{T}} \mathbf{r}^{*}\right] \\
& =\frac{1}{2}\left[\begin{array}{ll}
\mathbf{m}^{\mathcal{H}} & \mathbf{m}^{\mathcal{T}}
\end{array}\right]\left[\begin{array}{c}
\mathbf{r} \\
\mathbf{r}^{*}
\end{array}\right]=\frac{1}{2}\left[\begin{array}{c}
\mathbf{m} \\
\mathbf{m}^{*}
\end{array}\right]^{\mathcal{H}}\left[\begin{array}{c}
\mathbf{r} \\
\mathbf{r}^{*}
\end{array}\right]=\frac{1}{2} \mathbf{w}^{\mathcal{H}} \mathbf{y} .
\end{aligned}
$$

Similarly, $\operatorname{Re}\left\{\mathbf{m}^{\mathcal{H}} \mathbf{s}_{1}\right\}$ can be expressed as $\operatorname{Re}\left\{\mathbf{m}^{\mathcal{H}} \mathbf{s}_{1}\right\}=$ $\frac{1}{2} \mathbf{w}^{\mathcal{H}} \mathbf{s}_{1}^{\prime}$, where $\mathbf{s}_{1}^{\prime}=\left[\begin{array}{ll}\mathbf{s}_{1} & \mathbf{s}_{1}^{*}\end{array}\right]^{\mathcal{T}}$. With the method of Lagrange multipliers, we obtain

$$
\begin{aligned}
\lambda(\mathbf{w})= & \mathrm{E}\left\{\left|\operatorname{Re}\left\{\mathbf{m}^{\mathcal{H}} \mathbf{r}\right\}-A_{1} b_{1}\right|^{2}\right\}-\mu\left(\mathbf{w}^{\mathcal{H}} \mathbf{s}_{1}^{\prime}-1\right) \\
= & \mathrm{E}\left\{\left(0.5 \mathbf{w}^{\mathcal{H}} \mathbf{y}-A_{1} b_{1}\right)\left(0.5 \mathbf{w}^{\mathcal{H}} \mathbf{y}-A_{1} b_{1}\right)^{*}\right\}-\mu\left(\mathbf{w}^{\mathcal{H}} \mathbf{s}_{1}^{\prime}-1\right) \\
= & 0.25 \mathbf{w}^{\mathcal{H}} \mathrm{E}\left\{\mathbf{y} \mathbf{y}^{\mathcal{H}}\right\} \mathbf{w}-0.5 A_{1} \mathbf{w}^{\mathcal{H}} \mathrm{E}\left\{\mathbf{y} b_{1}\right\}-0.5 A_{1} \mathrm{E}\left\{b_{1} \mathbf{y}^{\mathcal{H}}\right\} \mathbf{w} \\
& +A_{1}^{2}-\mu\left(\mathbf{w}^{\mathcal{H}} \mathbf{s}_{1}^{\prime}-1\right) \\
= & 0.25 \mathbf{w}^{\mathcal{H}} \mathbf{C}_{\mathbf{y y}} \mathbf{w}-\left(0.5 A_{1}^{2}+\mu\right) \mathbf{w}^{\mathcal{H}} \mathbf{s}_{1}^{\prime}-0.5 A_{1} \mathbf{s}_{1}^{\prime \mathcal{H}} \mathbf{w}+\left(A_{1}^{2}+\mu\right)
\end{aligned}
$$

where $\mu$ is the Lagrange multiplier and

$$
\begin{aligned}
\mathbf{C}_{\mathbf{y y}} & =\mathrm{E}\left\{\mathbf{y} \mathbf{y}^{\mathcal{H}}\right\}=\mathrm{E}\left\{\left[\begin{array}{c}
\mathbf{r} \\
\mathbf{r}^{*}
\end{array}\right]\left[\begin{array}{ll}
\mathbf{r}^{\mathcal{H}} & \mathbf{r}^{\mathcal{T}}
\end{array}\right]\right\} \\
& =\mathrm{E}\left\{\left[\begin{array}{cc}
\mathbf{r} \mathbf{r}^{\mathcal{H}} & \mathbf{r r}^{\mathcal{T}} \\
\mathbf{r}^{*} \mathbf{r}^{\mathcal{H}} & \mathbf{r}^{*} \mathbf{r}^{\mathcal{T}}
\end{array}\right]\right\}=\left[\begin{array}{ll}
\mathbf{C}_{\mathbf{r r}} & \tilde{\mathbf{C}}_{\mathbf{r r}} \\
\tilde{\mathbf{C}}_{\mathbf{r r}}^{*} & \mathbf{C}_{\mathbf{r r}}^{*}
\end{array}\right]
\end{aligned}
$$

Differentiating $\lambda(\mathbf{w})$ with respect to $\mathbf{w}$ yields

$$
\frac{\partial \lambda(\mathbf{w})}{\partial \mathbf{w}}=0.25\left(\mathbf{C}_{\mathbf{y y}} \mathbf{w}\right)^{*}-0.5 A_{1} \mathbf{s}_{1}^{\prime *}
$$

The above equation holds since

$$
\begin{aligned}
& \frac{\partial \mathbf{w}^{\mathcal{H}} \mathbf{C}_{\mathbf{y y}} \mathbf{w}}{\partial \mathbf{w}}=\left(\mathbf{C}_{\mathbf{y y}} \mathbf{w}\right)^{*}, \\
& \frac{\partial \mathbf{w}^{\mathcal{H}} \mathbf{s}_{1}^{\prime}}{\partial \mathbf{w}}=\mathbf{0} ; \quad \frac{\partial \mathbf{s}_{1}^{\prime \mathcal{H}} \mathbf{w}}{\partial \mathbf{w}}=\mathbf{s}_{1}^{\prime *} .
\end{aligned}
$$

Setting (9) to zero yields the optimum detector for the first user

$$
\mathbf{w}_{1}=2 A_{1} \mathbf{C}_{\mathbf{y y}}^{-1} \mathbf{s}_{1}^{\prime}
$$


By performing eigen-decomposition of $\mathbf{C}_{\mathbf{y y}}$, we obtain

$$
\mathbf{C}_{\mathbf{y y}}=\mathbf{U}_{s}^{\prime} \Lambda_{s}^{\prime} \mathbf{U}_{s}^{\prime \mathcal{H}}+\mathbf{U}_{n}^{\prime} \Lambda_{n}^{\prime} \mathbf{U}_{n}^{\prime \mathcal{H}}
$$

where $\mathbf{U}_{s}^{\prime}$ is $2(N+L-1) \times(2 P+1) K$ matrix whose columns are the eigenvectors of the signal subspace, and $\Lambda_{s}^{\prime}$ is a $(2 P+1) K \times(2 P+1) K$ diagonal matrix whose diagonal elements are the corresponding eigenvalues; while the columns of $\mathbf{U}_{n}^{\prime}$ are eigenvectors of the noise subspace, and $\Lambda_{n}^{\prime}$ contains the corresponding eigenvalues. The improved blind MMSE detector is derived by substituting (11) into (10)

$$
\mathbf{w}_{1}=2 A_{1}^{2} \mathbf{U}_{s}^{\prime} \Lambda_{s}^{\prime-1} \mathbf{U}_{s}^{\prime \mathcal{H}} \mathbf{s}_{1}^{\prime} .
$$

The above equation holds since $\mathbf{U}_{n}^{\prime \mathcal{H}} \mathbf{s}_{1}^{\prime}=\mathbf{0}$, i.e., $\mathbf{s}_{1}^{\prime}$ belongs to the signal subspace, and is orthogonal to the noise subspace.

Note that with the conventional linear MMSE algorithm expressed by (7), the detector is derived only using the subspace components obtained from the autocorrelation of the observation $\mathbf{C}_{\mathbf{r r}}$. The pseudo-autocorrelation matrix $\tilde{\mathbf{C}}_{\mathbf{r r}}$ is implicitly assumed to be zero. In the case of transmitting real-valued data over complex-valued channel, the pseudo-autocorrelation matrix $\tilde{\mathbf{C}}_{\mathbf{r r}}$ is non-vanishing [22] and utilization of the improperness property of the received signal will consequently lead to a better performance.

In practice, the eigen-components of the sample autocorrelation and pseudo-autocorrelation matrices based on $n$ received signal vector

$$
\mathbf{C}_{\mathbf{r r}} \approx \frac{1}{n} \sum_{i=1}^{n} \mathbf{r}(i) \mathbf{r}^{\mathcal{H}}(i) ; \quad \tilde{\mathbf{C}}_{\mathbf{r r}} \approx \frac{1}{n} \sum_{i=1}^{n} \mathbf{r}(i) \mathbf{r}^{\mathcal{T}}(i)
$$

are used for constructing the subspace-based blind detector. The longer batch length $n$, the more accurate estimates of $\mathbf{C}_{\mathbf{r r}}$ and $\tilde{\mathbf{C}}_{\mathbf{r r}}$, but at the same time, longer processing delays.

\section{NumericAl RESUlts}

Simulation results are presented in this section to demonstrate the effectiveness of the proposed scheme. In our simulations, user 1 is assumed to be the desired user. The spreading codes are randomly generated and normalized, and the spreading gain is $\mathrm{N}=31$. The complx channel coefficients of each user are randomly generated and normalized and have exponential decay profile. We consider balanced transmission, i.e., the amplitude $A_{k}$ is equal for all the users. Perfect knowledge of the first user's channel impulse response is assumed in our simulations. During each Monte-Carlo run, the block size is set to 50000 bits, of which $n=5000$ are used to estimate the signal subspace. All presented results are averaged over at least 100 Monte Carlo simulations.

In Fig. 1, we compare the performance of the conventional blind MMSE detector and the proposed blind MMSE detectors at different SNR levels for a $K=10$ user system in a $L=11$ path channel. One can see from the figure that the proposed blind detector achieves a gain up to $1 \mathrm{~dB}$ compared to the conventional blind detector. As will become evident later on, further gain is attainable by increasing the batch length $n$. The performance gap tends to increase as the SNR level increases.

The impact of the batch length $n$ for subspace estimation is examined in Fig 2, where the parameters are set to be $K=$

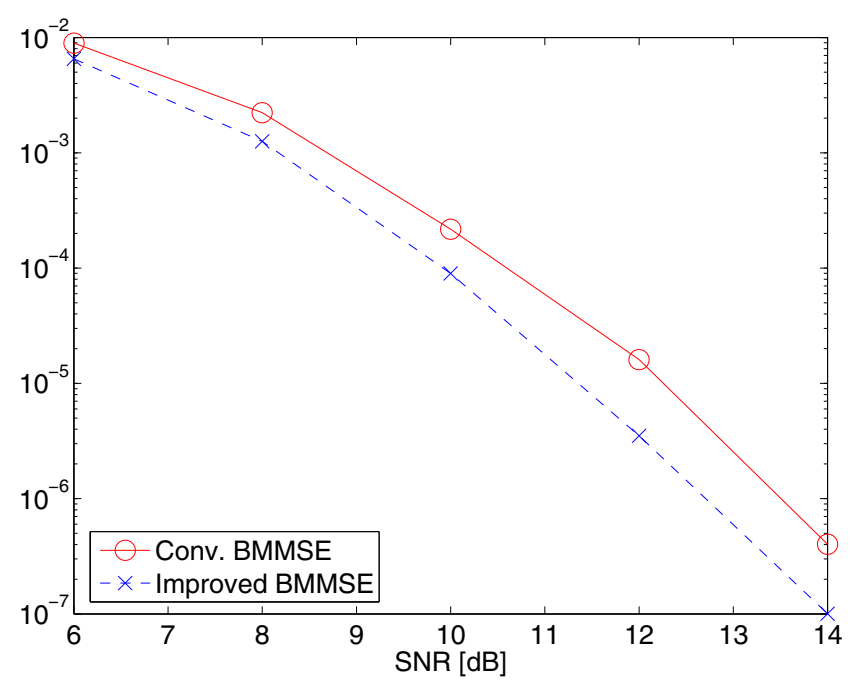

Fig. 1. Performance comparison in multipath fading channels $(K=10, L=$ $11, n=5000)$.

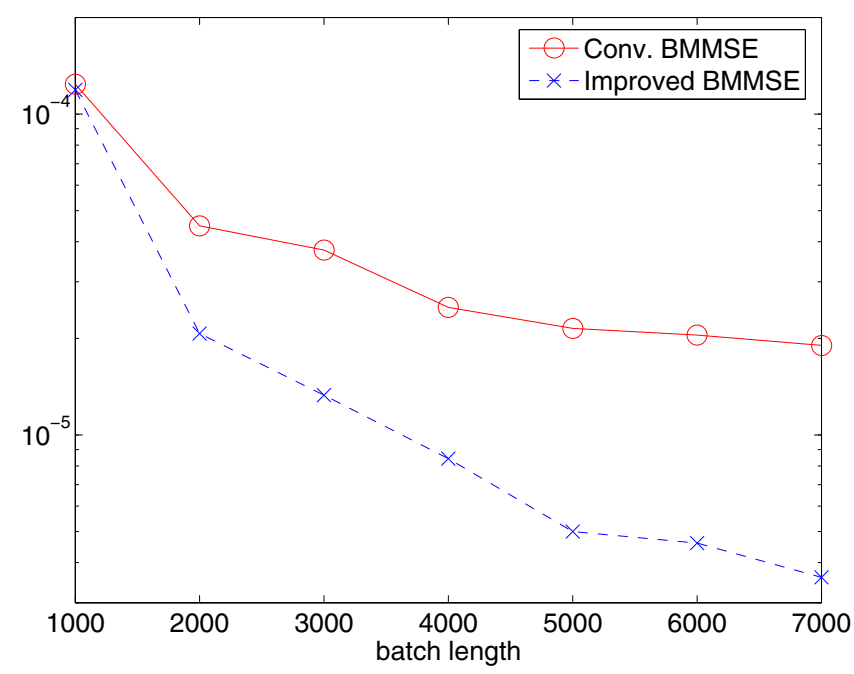

Fig. 2. BER vs. batch length for subspace estimation $(K=10, L=$ $\left.11, E_{b} / N_{0}=12 \mathrm{~dB}\right)$.

$10, L=11, E_{b} / N_{0}=12 \mathrm{~dB}$. The performance of the conventional detector tends to get saturated after the batch length goes beyond 4000 , whereas the performance of the proposed detector continues to improve beyond this point.

The system capacity, i.e., the number of users that can be supported by a system with acceptable BER, for the two detectors, is examined in Fig. 3. The number of multipath and SNR value are set to be $L=28$ and $E_{b} / N_{0}=12 \mathrm{~dB}$, respectively. Apparently, the proposed detector increases the system capacity significantly compared to the conventional detector. For instance, with a target BER $=10^{-4} / 10^{-5}$, around $18 / 13$ users can be supported by the proposed scheme, whereas only $14 / 9$ users can be supported by the conventional scheme. Another important discovery is that the performance gap between the two schemes becomes more obvious as the number of users increases, meaning that it is more advantageous to apply the proposed blind detection scheme in a heavily loaded system. 


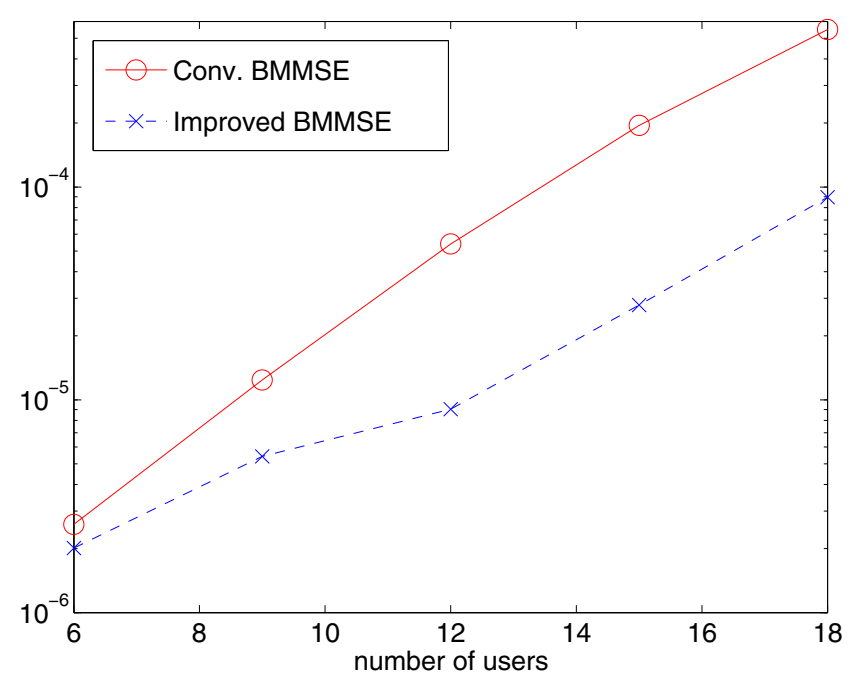

Fig. 3. Comparison of system capacity $\left(L=28, n=5000, E_{b} / N_{0}=12\right.$ $\mathrm{dB})$.

\section{Conclusions}

A new interference mitigation scheme based on signal subspace estimation is developed for DS-CDMA systems in frequency-selective channels. The blind algorithm proposed in this paper only requires the knowledge of the signature waveform and timing of the desired users. It does not need any training nor require the spreading codes and time delays of the other users. Its simplicity makes it attractive for mobile units as well as the centralized detector in a based station of a cellular mobile network. Comparing to the conventional subspace-based blind multiuser detector, the proposed blind detector achieves a superior performance by exploiting the improper nature of MAI and ISI. We also show that the performance advantages obtained by applying the proposed scheme increases when the number of active users becomes larger, which makes it a preferred choice for heavily loaded DS-CDMA systems.

\section{REFERENCES}

[1] S. Verdu. Multiuser Detection. Cambridge University Press, 2001

[2] R. Lupas and S. Verdu. "Linear multiuser detector for synchronous codedivision multipleaccess channels", IEEE Trans. Inform. Theory, vol. 35 , pp. 123-136, 1989.

[3] R. Lupas and S. Verdu, "Near-far resistance of multiuser detectors in asynchronous channels", IEEE Trans Commun., vol. 38, pp. 496-508, 1990

[4] Z. Xie, R.T. Short and C.K. Rushforth. "A family of suboptimum detectors for coherent multiuser communications", IEEE J. Select. Areas Commun., vol.8, no. 4, pp. 683 - 690, 1990

[5] U. Madhow, M. Honig. "MMSE interference suppression for directsequence spread-spectrum CDMA", IEEE Trans. Commun., vol. 42, no. 12, pp. 3178-3188, 1994.

[6] M. Honig, U. Madhow, S. Verdu. "Blind adaptive multiuser detection", IEEE Trans. Inform. Theory, vol. 41, no. 4, pp. 944-960, July 1995

[7] X. Wang and H.V. Poor. "Blind multiuser detection: a subspace approach", IEEE Trans. Inform. Theory, vol. 44, no. 2, pp. 677-690, 1998.

[8] X. Wang and H.V. Poor. "Blind equalization and multiuser detection in dispersive CDMA channels", IEEE Trans. Commun., vol. 46, no. 1, pp. 91-103, 1998.

[9] J. Namgoong, T. Wong, J. Lehnert. "Subspace multiuser detection for multicarrier DS-CDMA”, IEEE Trans. on Commun., pp. 1897-1908, Nov. 2000.

[10] J. Proakis. Digital Communications. McGraw-Hill Book Co, Singapore, 2001, 4th edition

[11] X. Wang and H.V. Poor. "Blind adaptive multiuser detection in multipath CDMA channels based on subspace tracking", IEEE Trans. Signal Process., vol. 46, no. 11, pp. 3030-3044, 1998.
[12] H. Zhou, P. Xiao, W. Woo, B. Sharif. "Expanded decorrelating detector with reduced noise enhancement for multipath frequency-selective fading channels", IET Commun., In press.

[13] S. Buzzi, M. Lops, A. Tulino "A new family of MMSE multiuser receivers for interference suppression in DS/CDMA systems employing BPSK modulation". IEEE Trans. on Commun., vol. 49, no. 1, pp. 154 167, Jan. 2001.

[14] A. Mirbagheri, K. Plataniotis, S. Pasupathy. "An enhanced widely linear CDMA receiver with OQPSK modulation", IEEE Trans. on Commun. vol. 54, no. 2, pp. 261-272, Feb. 2006.

[15] Y. Yoon, H. Kim. "An efficient blind multiuser detection for improper DS/CDMA signals”. IEEE Trans. on Veh. Technol., vol. 55, no. 2, pp. 572-582, March 2006.

[16] W. Gerstacker, F. Obernosterer, R. Schober, A. Lehmann, A. Lampe, P. Gunreben. "Equalization concepts for Alamouti's space-time block code", IEEE Trans. on Commun., vol. 52, no. 7, pp. 1178-1190, July 2004.

[17] M. Witzke, S. Baro, J. Hagenauer. "Iterative detection of generalized coded MIMO signals using a widely linear detector", Proc. IEEE Globecom, vol. 4, pp. 1821-1825, Dec. 2003.

[18] A. Lampe, R. Scholber, W. Gerstacker, J. Huber. "A novel iterative multiuser detector for complex modulation schemes", IEEE J. Select. Areas Commun., vol. 20, no. 2, pp. 339-350, Feb. 2002.

[19] P. Schreier, L. Scharf, C. Mullis. "Detection and estimation of improper complex random signals", IEEE Trans. on Inform. Theory, vol. 51, no. 1, pp. 306-312, January 2005.

[20] F. Neeser, J. Massey. "Proper complex random processes with applications to information theory", IEEE Trans. on Inform. Theory, vol. 39, no. 4, pp. 1293-1302, July 1993.

[21] B. Picinbono, P. Chevalier. "Widely linear estimation with complex data", IEEE Trans. on Signal Process., vol. 43, no. 8, pp. 2030-2033, August 1995.

[22] W. Gerstacker, R. Schober, A. Lampe. "Receivers with widely linear processing for frequency-selective channels". IEEE Trans. on Commun., vol. 51, no. 9, pp. 1512-1523, Sept. 2003. 